\title{
Wavejets: A Local Frequency Framework for Shape Details Amplification
}

\author{
Yohann Béarzi Julie Digne Raphaëlle Chaine \\ LIRIS, CNRS, Université Claude Bernard Lyon 1
}

\begin{abstract}
Detail enhancement is a well-studied area of $3 D$ rendering and image processing, which has few equivalents for $3 D$ shape processing. To enhance details, one needs an efficient analysis tool to express the local surface dynamics. We introduce Wavejets, a new function basis for locally decomposing a shape expressed over the local tangent plane, by considering both angular oscillations of the surface around each point and a radial polynomial. We link the Wavejets coefficients to surface derivatives and give theoretical guarantees for their precision and stability with respect to an approximate tangent plane. The coefficients can be used for shape details amplification, to enhance, invert or distort them, by operating either on the surface point positions or on the normals. From a practical point of view, we derive an efficient way of estimating Wavejets on point sets and demonstrate experimentally the amplification results with respect to noise or basis truncation.
\end{abstract}

Categories and Subject Descriptors (according to ACM CCS): I.3.5 [Computer Graphics]: Computational Geometry and Object Modeling-Curve, surface, solid and object representations

\section{Introduction}

Many shape processing methods, whether they target shape segmentation, shape denoising or shape editing, rely heavily on surface derivatives estimates. Surface derivatives are indeed useful to estimate important shape features such as normals or curvatures. The signal processing viewpoint is slightly different: instead of analyzing signal derivatives, signals are often processed by using a frequency analysis and by devising filters operating on the Fourier coefficients. In this paper, we propose to bring together these two trends in a new function basis taking into account both the local surface derivatives and the angular oscillations of the surface around each point of the surface. We consider surfaces as smooth manifolds that can locally be expressed as a height field over a planar parameterization. In this setting, we locally analyze the surface by extending the osculating Jets [CP03] in order to take into account both the local angular oscillation frequencies, and their evolution with respect to an increasing radius. This formulation, which we term Wavejets, gives valuable information on the shape by emphasizing that the local behavior of the surface along an arbitrary radial direction from $\boldsymbol{p}$ is a polynomial function of the distance to $\boldsymbol{p}$ in the parameterization plane. We propose to compute the Wavejets using the tangent plane for parameterization purpose and we demonstrate some theoretical properties of the Wavejets. In particular, we quantify the stability of the decomposition for a small deviation of the parameterization plane with respect to the tangent plane. This result is useful for estimating Wavejets on surfaces described by point sets. Furthermore, Wavejets coefficients can be used to compute in- teresting indicators of differential volumes that can be an alternative to using curvatures and further surface derivatives. Those indicators are used to devise efficient surface filters. We demonstrate two applications of these filters working directly on point sets: position filtering for detail modification (such as detail amplification or detail inversion) and normal modification. Figure 1 shows an example of detail amplification on the Armadillo shape.

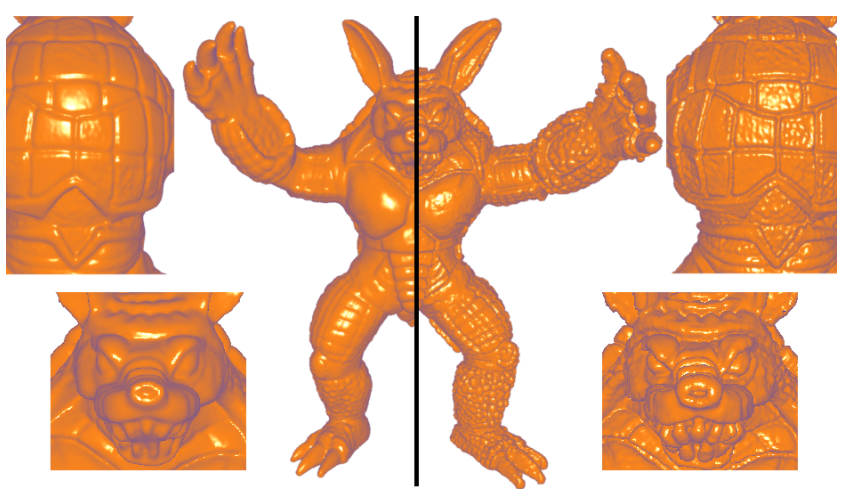

Figure 1: Amplifying details of the Armadillo shape with a Wavejets filter.

To summarize, our contributions are:

- A local frequency framework for representing a surface, whose precision and stability are proven.

- A practical method for computing Wavejets for point set surfaces 
- Efficient shape processing filters for detail amplification, inversion and warping using Wavejets coefficients.

\section{Related work}

Surface derivatives estimation. Computing surface derivatives has raised a lot of work in the geometry processing community. [CP03] introduced the notion of osculating $n$-jets to analyze a surface around a point. A $n$-jet is a truncated Taylor expansion used to locally estimate a smooth surface. Each monomial coefficient of a Taylor expansion is directly linked to a high order derivative of the surface, which yields information about the normal, local curvature, or higher order differential quantities. By fitting an osculating jet of order $K$ to a set of surface points in a neighborhood of radius $r$, the precision of all $k$ order derivatives is $o\left(r^{K-k}\right)$. [MT98] first introduced a frequency interpretation of the local variations on a surface. Given the principal curvatures at one point, a second order smoothness measure is defined as the integral over a circle of a second order polynomial. A similar process is proposed to define a third order smoothness measure. Following this work, [JS10] proposed to interpret third order derivatives of a surface as Fourier series coefficients of the height function above a circle in the tangent plane. Interpreting high order derivatives as Fourier coefficients is interesting since it makes the choice of the origin vector of the local parameterization plane irrelevant.

In many cases, however, surfaces are known only through a set of discrete and potentially noisy measures obtained by a 3D acquisition system. Local surface derivatives are directly impacted by this noise since the noise is amplified through an explicit derivation process. To alleviate this effect, one can compute some local differential quantities by integration. Integral invariants build on this principle to define principal curvatures based descriptors $\left[\mathrm{MCH}^{*}\right.$ 06, $\mathrm{PWY}^{*}$ 07, PWHY09]. Integral invariants can be computed directly on meshes or on grids, or on point sets using local surface regression or interpolation. Similarly, [DM14] used covariance analysis to compute principal directions and curvatures of point sets in an asymptotically consistent way. On meshes, the derivatives can be estimated by considering the provided connectivity [MDSB03, WMKG07]. Finally, it is also possible to use metrics derived from heat diffusion to give new expressions of surface derivatives [LSW09].

Signal Processing-like approaches. Many approaches have tackled the problem of surface filtering by mimicking standard signal processing algorithms [PG01]. [Tau95] noticed that Fourier basis functions are eigenfunctions of the Laplacian operator on $\mathbb{R}^{2}$. Thus, by building a Laplacian operator over a surface and extracting its eigenfunctions, the projection of the point coordinates functions on such a basis gives a spectral decomposition which can be used for designing low pass filters [Tau95, TZG96]. Pauly proposed to use iterative Laplacian smoothing to separate the signal into high and low frequency information and designs Fourier-like filters in this setting [PKG06]. Defining a Laplacian operator on a mesh has been widely discussed. [VL08] proposed a formulation yielding an orthogonal basis: the Manifold Harmonics Basis. Spectral processing can be directly performed in this basis as if it was a Fourier basis, hence low-pass, high-pass or high-boost filtering has a straightforward implementation, provided the shape eigenvectors are known. Spherical Harmonics were also explored as a way to get a spectral decomposition of a shape parameterized on a sphere in a rotation-invariant way [KFR03]. Spherical harmonics are restricted to genus 0 shapes and are a global basis of the shape, but they can be used to design high- or low-pass filters [ZBS04] or for detail transfer [MCAG08]. In this paper, we set up a framework for the local decomposition of a surface using a new basis. This basis is tailored for analyzing the surface around one point, and for computing differential quantities at that point. Decomposing the signal on this meaningful function basis is the core of Wavejets. Wavejets are therefore related to Zernike basis functions [Zer34], an orthogonal basis, introduced for optical lenses analysis and used often for processing images (e.g. [KH90]) or shape retrieval [NK03]. Although a linear relationship exists between Wavejets and Zernike polynomials, the latter do not give direct access to differential quantities, which are provided by our Wavejets basis by construction.

Detail Exaggeration. While the literature for detail exaggeration in image or video processing is large (e.g. [LTF* 05, DMIF15]) it has been far less studied for surfaces. Existing methods can be sorted out in two categories: the first one modifies the rendering of a shape while the second modifies the shape through its normals or point positions to enhance the shape details. Modifying the rendering of a shape to enhance the details can be considered as a non-photorealistic rendering method in the special case where the purpose is to amplifly the details. Rusinkiewicz et al. [RBD06] propose to modify the shader in a multiscale way and merge this multiscale information with the classic shading color. Detail Amplification through rendering has been also studied via view-dependent feature computation [VPB* 09 ]. The second category of approaches explicitly modifies the shape information in a viewpoint-independent manner. In image processing the equivalent operation is done by the so-called High Boost Filter, also known as Unsharp Masking [PRM00, MLLY91, RSKMY96], which moves each pixel value in a direction opposite to the one of the Laplacian smoothing, to sharpen the contrasts. This idea dates back to Gabor [Gab65, LFB94], and is known to produce artifacts if the filter is iterated too many times. This filter can be trivially adapted to point sets, and we will compare our method to it. Another way of amplifying the visualization of details is to modify only the normals at the points. Cignoni et al. [CST05] propose to modify the normals iteratively in a direction opposite to the mean normal of the neighbors. This process can seen as a high boost filter applied to the normals. Finally, as a side-benefit of Algebraic Point Set Surfaces rendering [GGG08], a curvature parameter controls the detail enhancement or inversion, a feature that can also be obtained in our Wavejets framework.

\section{Wavejets}

\subsection{Definition}

Osculating jets, originally introduced in [CP03] are high order polynomials that correspond to local truncated Taylor expansions of height field surfaces, thus providing interesting differential properties, such as the normal and principal curvatures, for smooth surfaces. We introduce a different kind of jets, named Wavejets, that 


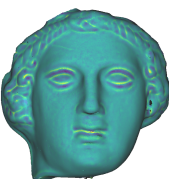

$\phi_{0,0}$

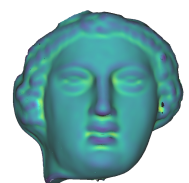

$\phi_{2,0}$

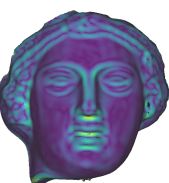

$\left|\phi_{2,2}\right|$

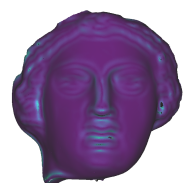

$\left|\phi_{3,1}\right|$

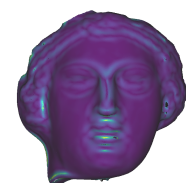

$\left|\phi_{3,3}\right|$

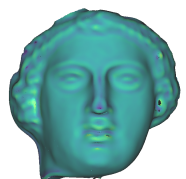

$\phi_{4,0}$

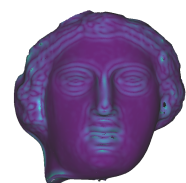

$\left|\phi_{4,2}\right|$

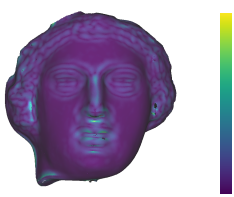

$\left|\phi_{4,4}\right|$

Figure 2: Computation of some values of $\phi_{k, n}(\boldsymbol{p})$ for a radius $R_{\phi}$ corresponding to the height of the eyes of the statue. If $k$ and $n$ do not share the same parity, coefficient $\phi_{k, n}$ is zero. For $k \geq 0$ and $n \neq 0$, only the magnitude of $\phi_{k, n}$ is displayed since the phase depends on the origin direction of the angles. For $k \geq 0, \phi_{k, 0}$ is real, does not depend on the origin direction, and can be negative. Note that $\phi_{1,1}(\boldsymbol{p})=0$ if the parameterization plane is the tangent plane at $\boldsymbol{p}$ to the surface. $\phi_{0,0}(\boldsymbol{p})$ measures the offset of the input point $\boldsymbol{p}$ to the surface which can be nonzero in the practical case where the Wavejets order is too low to catch every local variation around $p$.

retain all nice properties of the osculating Jets but also provide a natural interpretation in terms of local angular oscillations.

Let $\mathcal{S}$ be a smooth surface and $p$ a point on $\mathcal{S}$. As a consequence, the surface can be locally parameterized as a height field $f(x, y)$ inside a neighborhood of radius $R_{\phi}$ on a plane $\mathcal{P}(\boldsymbol{p})$ passing through $\boldsymbol{p}$. The neighborhood of $\boldsymbol{p}$ can be expressed as a Taylor Expansion:

$$
f(x, y)=\sum_{k=0}^{\infty} \sum_{j=0}^{k} \frac{f_{x^{k-j} y_{j}}(0,0)}{(k-j) ! j !} x^{k-j} y^{j}
$$

where $f_{x^{k-j} y^{j}}=\frac{\partial^{k} f}{\partial x^{k-j} \partial y^{j}}$.

Restricting to a circle of radius $r\left(r<R_{\phi}\right)$ centered at $\boldsymbol{p}$ in $\mathcal{P}(\boldsymbol{p})$, and expressing $f$ with respect to an angle $\theta$ yields a periodic function $\theta \rightarrow f(r, \theta)$. $\theta$ is measured with respect to an arbitrary origin direction for the phases in $\mathcal{P}(\boldsymbol{p})$.

Using polar coordinates $(r, \theta)$ with $(x, y)=(r \cos \theta, r \sin \theta)$ in equation 1 and Euler's formulas to express $\cos (n \theta)$ and $\sin (n \theta)$ as polynomials of $\cos \theta$ and $\sin \theta$, one can show that

$$
f(r, \theta)=\sum_{k=0}^{\infty} \sum_{n=-k}^{k} r^{k} \phi_{k, n} e^{i n \theta}=\sum_{n=-\infty}^{\infty} \sum_{k=|n|}^{\infty} r^{k} \phi_{k, n} e^{i n \theta}
$$

with $\phi_{k, n}=\sum_{j=0}^{k} \frac{1}{j !(k-j) !} b(k, j, n) f_{x^{k-j} y^{j}}(0,0) . b(k, j, n)$ is defined as follows :

- $b(k, j, n)=0$ if $k$ and $n$ do not have the same parity

- $b(k, j, n)=\frac{1}{2^{k} \boldsymbol{i}^{j}} \sum_{h=0}^{\frac{n-k}{2}}\left(\begin{array}{c}k-j \\ h\end{array}\right)\left(\begin{array}{c}j \\ \frac{n-k}{2}-h\end{array}\right)(-1)^{h}$ otherwise (see the supplementary material for the full derivation).

This amounts to decomposing the function on a new function basis $B_{k, n}(r, \theta)=r^{k} e^{i n \theta}$.

In other words, while osculating jets provide arbitrary high order derivatives $f_{x^{k-j} y^{j}}(0,0)$, our representation provides arbitrary high order Fourier coefficients $\phi_{k, n}(f)$ which are a linear combination of high order derivatives, combining them in a certain way that favors the independence of the coefficients with respect to the origin direction for the phases. More precisely, a rotation of the origin vector in the parameterization plane $\mathcal{P}(\boldsymbol{p})$ induces a phase shift of the coefficients. Figure 2 shows the amplitude of the first Wavejets terms $\phi_{k, n}$. Each $\phi_{k, n}$ is related to an order of radial derivation $k$ and to a number of oscillations $n$. A Wavejet of order $K$ is called a K-Wavejet.

\subsection{Properties}

Curvatures By explicitly writing the link between $\phi_{k, n}$ and the derivatives of $f$, the mean curvature $H(\boldsymbol{p})$ and the Gaussian curvature $K(\boldsymbol{p})$ at $\boldsymbol{p}$ can be obtained easily.

$$
\begin{gathered}
\phi_{1,1}=\phi_{1,-1}^{*}=\frac{1}{2}\left(f_{x}+\boldsymbol{i} f_{y}\right) \\
\phi_{2,0}=\frac{1}{2}\left(f_{x x}+f_{y y}\right) ; \phi_{2,2}=\phi_{2,-2}^{*}=\frac{1}{4}\left(f_{x x}-f_{y y}+\boldsymbol{i} f_{x y}\right)
\end{gathered}
$$

Since the Gaussian curvature $K(\boldsymbol{p})$ can be expressed w.r.t. partial derivatives of $f$ at $\boldsymbol{p}$ as $K(\boldsymbol{p})=\frac{f_{x x} f_{y y}-f_{x y}^{2}}{\left(1+f_{x}^{2}+f_{y}^{2}\right)^{2}}$, we get:

$$
K(\boldsymbol{p})=\frac{4 \phi_{2,0}^{2}-16 \phi_{2,-2} \phi_{2,2}}{\left(1+4 \phi_{1,-1} \phi_{1,1}\right)^{2}}
$$

Similarly, the mean curvature is expressed as $H(\boldsymbol{p})=$ $\frac{\left(1+f_{x}^{2}\right) f_{x x}+\left(1+f_{y}^{2}\right) f_{y y}-2 f_{x} f_{y} f_{y y}}{2\left(1+f_{x}^{2}+f_{y}^{2}\right)^{\frac{3}{2}}}$, yielding:

$$
H(\boldsymbol{p})=\frac{2 \phi_{2,0}\left(1+4 \phi_{1,-1} \phi_{1,1}\right)+4 \phi_{2,-2} \phi_{1,1}^{2}+4 \phi_{2,2} \phi_{1,-1}^{2}}{\left(1+4 \phi_{1,-1} \phi_{1,1}\right)^{\frac{3}{2}}}
$$

If $\mathcal{P}(\boldsymbol{p})=\mathcal{T}(\boldsymbol{p})$, the tangent plane to $\mathcal{S}$ at $\boldsymbol{p}$, then $\phi_{1,1}=\phi_{1,-1}=$ 0 , and :

$$
K(\boldsymbol{p})=4\left(\phi_{2,0}^{2}-4 \phi_{2,-2} \phi_{2,2}\right), H(\boldsymbol{p})=2 \phi_{2,0}
$$

The principal directions can be found using Wavejets by considering the signal $\sum_{n=-2}^{2} \phi_{2, n} e^{i n \theta}$. This signal contains a constant component $\phi_{2,0}$ and a component that oscillates two times and whose maximum is aligned with the first principal curvature direction (corresponding to the phase of $\phi_{2,2}$ ). As a consequence, the principal curvatures $\kappa_{1}$ and $\kappa_{2}$ can also be recovered using Wavejets:

$$
\kappa_{1}=2\left(\phi_{2,0}+\phi_{2,2}+\phi_{2,-2}\right) \text { and } \kappa_{2}=2\left(\phi_{2,0}-\phi_{2,2}-\phi_{2,-2}\right)
$$

\subsection{Stability}

We now turn to our main result for the stability of the Wavejets coefficients when the parameterization plane is close but different from the tangent plane. Let us call $\mathcal{T}(\boldsymbol{p})$ the true tangent plane and $\mathcal{P}(\boldsymbol{p})$ the chosen parameterization plane, also passing through $\boldsymbol{p}$. Since $\boldsymbol{p}$ belongs to both planes, they intersect along a 
line $\mathcal{T}(\boldsymbol{p}) \cap \mathcal{P}(\boldsymbol{p})$ of direction $u$. We consider the angle $\gamma$ such that the rotation of axis $(\boldsymbol{p}, u)$ and angle $\gamma$ transforms $\mathcal{P}(\boldsymbol{p})$ into $\mathcal{T}(\boldsymbol{p})$. Let us parameterize $\mathcal{T}(\boldsymbol{p})$ and $\mathcal{P}(\boldsymbol{p})$ so that a point of the surface has coordinates $(x=r \cos \theta, y=r \sin \theta, h)$ over $\mathcal{T}(\boldsymbol{p})$ and $(x=$ $R \cos \Theta, y=R \sin \Theta, H)$ over $\mathcal{P}(\boldsymbol{p})$. Let us first assume that $\theta$ (resp. $\Theta)$ corresponds to the angular coordinate of a point on the surface with respect to $u$ in $\mathcal{T}(\boldsymbol{p})$ (resp. with $u$ in $\mathcal{P}(\boldsymbol{p})$ ). In this setting, the surface Wavejets decomposition at point $\boldsymbol{p}$ writes $\sum_{k=0}^{\infty} \sum_{n=-k}^{n=k}$ $\phi_{k, n} r^{k} e^{i n \theta}$ over $\mathcal{T}(\boldsymbol{p})$ and $\sum_{k=0}^{\infty} \sum_{n=-k}^{n=+k} \Phi_{k, n} R^{k} e^{i n \Theta}$ over $\mathcal{P}(\boldsymbol{p})$. Using the rotation of angle $\gamma$, we can express the $\Phi_{k, n}$ coefficients with respect to the $\phi_{k, n}$.

We will state our main theorem in this particular setting of origin vector for the phases. To generalize the theorem to an arbitrary origin vector for the angular coordinate $\theta$, recall that a rotation of angle $\mu$ of the origin vector in $\mathcal{T}(\boldsymbol{p})$ amounts to a phase shift $\mu$. Thus, one can always change the origin vector, compute the Wavejets coefficients $\phi_{k, n}$ and recover the Wavejets coefficients for origin direction $u$ as $\phi_{k, n} e^{i n \mu}$ (similar formulas hold for $\Phi_{k, n}$ and an origin vector change in $\mathcal{P}(\boldsymbol{p}))$.

Theorem 1 The coefficients $\Phi_{k, n}$ w.r.t to $\mathcal{P}(\boldsymbol{p})$ can be expressed with respect to the coefficients $\phi_{k, n}$ in the tangent plane $\mathcal{T}(\boldsymbol{p})$ as follows:

$$
\begin{aligned}
& \Phi_{0,0}=0 \\
& \Phi_{1,1}=\Phi_{1,-1}^{*}=\frac{\gamma}{2} e^{-i \frac{\pi}{2}}+o(\gamma) \\
& \Phi_{k, n}=\phi_{k, n}+\gamma F(k, n)+o(\gamma)
\end{aligned}
$$

where $F(k, n)$ is a function of the $\phi$ coefficients of order lower than $k$.

\section{Proof: see the supplementary material.}

Corollary 1 It follows from Theorem 1 that $\left|\Phi_{1,1}\right|=\frac{1}{2} \gamma+o(\gamma)$ and $\arg \left(\Phi_{1,1}\right)=\frac{\pi}{2}+o(\gamma)$. Thus if the rotation is small enough, the phase of $\Phi_{1,1}$ shifted by $\pi / 2$ in the plane $\mathcal{P}(\boldsymbol{p})$ corresponds to the axis of rotation $u$. Therefore, it is possible to correct the parameterization plane into the tangent plane by performing a rotation of $\mathcal{P}(\boldsymbol{p})$ along the axis $u$ with rotation angle $2\left|\Phi_{1,1}\right|$.

Proof: see the supplementary material.

\subsection{Error correction}

Corollary 2 One can recover the true coefficients $\phi_{k, n}$ iteratively, starting from the lowest order coefficients as:

$$
\begin{aligned}
& \phi_{k, n}=\Phi_{k, n}-\gamma \sum_{j=1}^{k-2} s_{j, k, n}+o(\gamma) \\
& s_{\substack{j, k, n \\
p+m=n \\
|p| \leq k-j \\
|m| \leq j}} \frac{\phi_{k-j, p}}{2 \boldsymbol{i}}\left(\phi_{j+1, m+1}(m+j+2)+\phi_{j+1, m-1}(m-j-2)\right)
\end{aligned}
$$

In particular, $\phi_{2,0}=\Phi_{2,0}+o(\gamma), \phi_{2,2}=\Phi_{2,2}+o(\gamma), \phi_{2,-2}=$ $\Phi_{2,-2}+o(\gamma)$, which means that the mean, Gaussian and principal curvatures are also stable in $o(\gamma)$.

Proof: see the supplementary material.

\subsection{Difference with Jets and Zernike}

There exists a linear map between Wavejets $\phi_{k, n}$, Jets $J_{k, j}$ [CP03] and Zernike polynomials $Z_{k}^{n}$ [Zer34]. This means that there is a way to compute any quantity equivalently from either representation as soon as the linear map is explicit. However this linear map is far from trivial and computing certain quantities will be easier using one or another function basis.

Jets give a direct expression of high-order cross derivatives of a surface. This representation is well-suited to tangent plane estimation, since the cross derivatives of order $1, J_{1,0}$ and $J_{1,1}$, are equal to 0 if the surface is parameterized with respect to the tangent plane. When fitting a jet to a set of surface points, the offset between the parameterization plane and the approximated surface is given directly by $J_{0,0}$.

Zernike polynomials give a polar representation of the surface. Zernike basis is orthogonal, which makes it easy to estimate the coefficients on regular polar grids. However, the error between a parameterization plane and the tangent plane is hard to express using Zernike coefficients. This error can be found as a non-trivial linear combination of $Z_{k}^{ \pm 1}$. Similarly, the error offset obtained when fitting Zernike polynomials to a set of points is given by a non-trivial linear combination of $Z_{k}^{0}$.

Wavejets give a representation which is mid-way between Jets and Zernike polynomials. Wavejets coefficients explicitly hold information about the tangent plane. When used to fit a set of surface points, they also provide a direct information on the offset between the surface and the parameterization plane. Besides, they split the surface into components corresponding to different angular frequencies in a similar manner as Zernike decomposition. This angular separation holds important information about the surface, which we will use to design our filters. In particular, we will provide some integral invariants by integration over angle $\theta$, which is straightforward using Wavejets or Zernike and difficult with the Jets. Finally, the error in the orientation of the tangent plane or the offset to the surface are easy to express using Wavejets and Jets but difficult using Zernike polynomials. Thus, Wavejets retain interesting properties from Jets and Zernike polynomials while avoiding some of their weaknesses.

\section{Details filtering and enhancement}

\subsection{Principle}

Let us consider the signed volume $\boldsymbol{V}(s)$ delimited by the surface and the parameterization plane in a small radius $s<R_{\phi}$ around a point $\boldsymbol{p}$. We can express $\boldsymbol{V}(s)$ as the sum of infinitesimal angular slices of the volume between the surface and the tangent plane: $\boldsymbol{V}(s)=\int_{0}^{2 \pi} A(\theta, s) d \theta$. In the plane corresponding to the angle $\theta$, $A(\theta, s)$ denotes the area enclosed between the surface and the tan- 


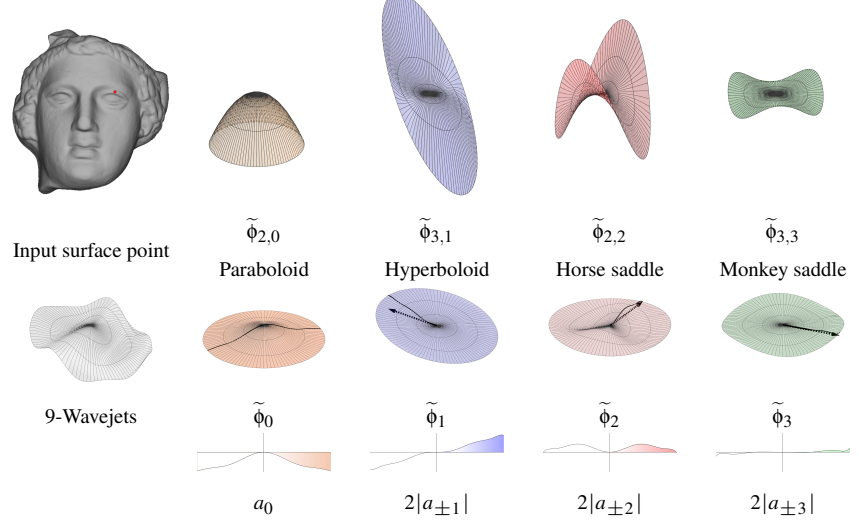

Figure 3: Wavejets decomposition around a point of a surface. Left: approximated 9-Wavejets surface. Let $\widetilde{\phi}_{k, n}(r, \theta)=$ $r^{k}\left(\phi_{k, n} e^{i n \theta}+\phi_{k,-n} e^{-i n \theta}\right)$ and $\widetilde{\phi}_{n}=\sum_{k=0}^{\infty} \widetilde{\phi}_{k, n}$. The amplitude of the functions $\widetilde{\phi}_{n}$ is more comparable to the amplitude of the input surface than the functions $\widetilde{\phi}_{k, n}$.

gent plane, for a radius varying between 0 and $s$, each point of the surface being scaled by the radius.

$$
\begin{aligned}
A(\theta, s) & =\int_{0}^{s}\left(\sum_{k=0}^{\infty} \sum_{n=-k}^{k} r^{k} \phi_{k, n} e^{i n \theta}\right) r d r \\
& =\sum_{n=-\infty}^{\infty}\left(\int_{0}^{s} \sum_{k=|n|}^{\infty} \phi_{k, n} r^{k+1} d r\right) e^{i n \theta}=\sum_{n=-\infty}^{\infty} a_{n}(s) e^{i n \theta}
\end{aligned}
$$

Coefficients $a_{n}(s)$ correspond to a Fourier decomposition of $A(\theta, s)$. By developping the corresponding integrals for the radius $s$, we obtain the following closed form:

$$
a_{n}(s)=\sum_{k=|n|}^{\infty} \frac{\phi_{k, n} s^{k+2}}{k+2}
$$

One can show that if $n \neq 0,2\left|a_{ \pm n}\right|(s)$ is the amplitude of the oscillating function $\int_{0}^{s} \widetilde{\phi}_{n}(r, \theta) r d r$, where $\widetilde{\phi}_{n}(r, \theta)$, as defined in Figure 3 , is the surface restricted to frequencies $\pm n$. For the special case $n=0, a_{0}(s)$ is the static part of $\int_{0}^{s} f(r, \theta) r d r$. Thus, $\forall \theta, a_{0}(s)=\int_{0}^{s} \widetilde{\phi}_{0}(r, \theta) r d r$ (see Figure 3).

Each coefficient $a_{n}(s)$ has interesting properties regarding the local surface dynamics. $2 \pi a_{0}(s)$ is equal to the signed volume between the surface and the parameterization plane. Indeed,

$$
\int_{0}^{2 \pi} \int_{0}^{s} f(r, \theta) r d r d \theta=\int_{0}^{2 \pi} A(\theta, s) d \theta=2 \pi a_{0}(s)
$$

Importantly enough, the $a_{n}$ are intrinsic quantities of the local surface neighborhood, and as such do not depend on the initial parameterization choice.

Remark 1 Equation (13) relates to the Volume Descriptor $V_{s}(\boldsymbol{p})$ introduced by $\left[\mathrm{MCH}^{*} 06\right]$ as follows:

$$
V_{s}(\boldsymbol{p})-2 \pi a_{0} \approx \frac{2}{3} \pi s^{3} .
$$

Thus, $a_{0}(s)$ measures the local deviation of the surface with regards to the tangent plane at $\boldsymbol{p}$. Local mean curvature is commonly used to reflect this local deviation, but it is meaningless in some cases, for example some points might have 0 mean curvature but nonzero higher orders derivatives and $a_{0}$. Such is the case of $(0,0)$ for $f(x, y)=x^{4}+y^{4}$. At those points, it is necessary to look at higher order derivatives to reveal the local dynamics of the surface. $a_{0}(s)$ involves higher order $\phi_{k, 0}$ with $k>0$, whereas local mean curvature is only proportional to $\phi_{2,0}$. In addition, the mean curvature is a measure per point, whose estimation precision from point sets is theoretically controlled by the polynomial order, therefore it will capture extremely small variations. $a_{0}(s)$ will on the contrary capture variations at a scale controlled by $s$.

Similarly, $a_{ \pm 1}(s)$ locally measures a first order antisymmetric tendency of the surface to change its normal direction when one moves away from $\boldsymbol{p}$, along the direction given by $\theta$. Here, this antisymmetric change corresponds to the variation of the tangent plane to be added on top of the variation induced by the mean variation of the position (reflected by $a_{0}(s)$ ). More precisely, the normal direction tends to change less when one moves away in the tangent plane in the direction orthogonal to the phase $\arg \left(a_{-1}(s)\right)$. Thus, $\arg \left(a_{-1}(s)\right)$ gives the direction in which the rotation of the tangent plane is maximal. In a nutshell, it measures around which direction and with what intensity the normal evolves. $\phi_{3, \pm 1}$ should theoretically be enough to give such information. However, similarly to $\phi_{2,0}, \phi_{3, \pm 1}$ is an infinitesimal measure whereas $a_{ \pm 1}(s)$ gives smoother information while still being able to catch high-order local variations thanks to the high-order estimation of the surface. Figure 3 illustrates the sensitivity of coefficients $\phi_{2,0}$ and $\phi_{3, \pm 1}$ compared to $a_{0}(s)$ and $a_{ \pm 1}(s)$ in the 9-Wavejets decomposition of a surface. Using the $a_{n}$ instead of the Wavejets coefficients themselves can also be seen as an extension of the use of averaged quantities to compute surface derivatives [ $\left.\mathrm{MCH}^{*} 06, \mathrm{PWY}^{*} 07, \mathrm{DM} 14\right]$.

To design the detail enhancement filters, we use Wavejets computed w.r.t. the tangent plane of the surface, and we only rely on $a_{0}(s)$ and $a_{ \pm 1}(s)$. Higher order values $a_{ \pm n}(s)$ would carry further information of higher order regarding the enclosed area in the direction $\theta$. For example, $a_{ \pm 2}(s)$ measures the tendency of the evolution of the local horse saddle shape, $a_{ \pm 3}(s)$ gives information about the evolution of the monkey saddle shape, and so on. The only limit to this computation is the precision for the coefficients when the surface is only known through a set of points and that Wavejets are fit, since, as demonstrated by [CP03], the precision of order $k$ derivatives is a $o\left(R_{\Phi}^{K-k}\right)$. In such cases, coefficient $a_{ \pm n}(s)$ is less interesting as $n$ grows.

\subsection{Position enhancement filter}

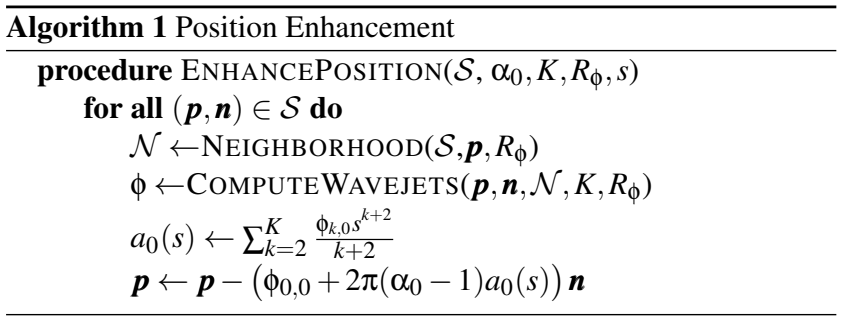


As noted previously, $a_{0}(s)$ gives the local deviation of the surface. If the volume, estimated by $2 \pi a_{0}(s)$ (See Equation 13), was to be increased, local details would be increased as well. Let $\alpha_{0}$ be the amplitude of the targeted detail enhancement. In order to increase the volume, a point $\boldsymbol{p}$ with normal $\boldsymbol{n}$ is moved to a new position $\boldsymbol{p}^{\prime}$ such that:

$$
\boldsymbol{p}^{\prime}=\boldsymbol{p}+\left(\phi_{0,0}-2 \pi\left(\alpha_{0}-1\right) a_{0}(s)\right) \boldsymbol{n}
$$

If $\alpha_{0}=1$, the motion is independent of $a_{0}(s)$ and the points are simply projected on the Wavejets surface. Indeed, when the Wavejets order is $K<\infty, \phi_{0,0}$ measures the distance between $\boldsymbol{p}$ and the truncated Wavejets surface. If the Wavejets approximation is exact, $\phi_{0,0}=0$. However, it is not the case in the presence of noise, on sharp edges, or in the approximation framework for point sets described in section 5 . The Wavejets surface is however considered as the true underlying surface and each point $\boldsymbol{p}$ is moved to this surface by displacing it by $\phi_{0,0}$. This has the effect of smoothening edges and canceling a part of the input noise. If $\alpha_{0}>1$, each point is first moved to the underlying surface and then moved proportionally to its underlying volume. Conversely, if $\alpha_{0}<0$, it will tend to invert the details and create anti-details.

The computation of $a_{0}(s)$ must be performed by using the tangent plane for parameterizing the surface at one point. Therefore, the parameterization plane should be corrected beforehand if it does not exactly fit the tangent plane as explained in section 3. Algorithm 1 assumes that this parameterization plane update has been performed. Notice that if the surface is smooth, the tangent plane and the enclosed volumes $a_{0}(s)$ evolve continuously over the surface. Therefore, the surface evolves continuously through the enhancement filter. In practice, this process cannot be applied to the infinite set of points on the continuous surface, however the filter definition remains valid in the continuous setting.

\subsection{Normal enhancement filter}

We propose a filter enhancing the details by exaggerating only the dynamics of the normals. Recall that $a_{ \pm 1}(s)$ measures the local balance of the shape by identifying the orientation (its phase) and the intensity (its absolute value) of the antisymmetric evolution of the tangent plane. The normal enhancement procedure amounts to modifying $\phi_{1, \pm 1}$ proportionally to $a_{ \pm 1}(s)$ at each point of the surface. The corresponding false normal is then estimated as the normal to a plane obtained by rotation of the parameterization following Corollary 1 . Notice that this false normal is not coherent with the real surface anymore. Given the desired detail normal evolution gain $\alpha_{1}=\alpha_{-1}^{*}$, the value of the new coefficient $\phi_{1, \pm 1}^{\prime}$ is defined as follows:

$$
\phi_{1, \pm 1}^{\prime}=-\pi\left(\alpha_{ \pm 1}-1\right) a_{ \pm 1}(s)
$$

Since $\phi_{1,1}=\phi_{1,-1}^{*}$, it is enough to compute either of these coefficients and deduce the false normal using Corollary 1.

Setting $\alpha=1$ leaves the surface unchanged. If $\alpha_{1}=2$, normals are enhanced, increasing the contrasts. If $\alpha_{1}=0$, the dynamic of the normals is totally compensated, and the surface looks smoothed out. If $\alpha_{1}<0$, normals are modified and "anti-details" appear in the rendering. $\alpha_{1}$ can also take imaginary values, which skew normals towards one direction as shown in figure 15 . Note that, when

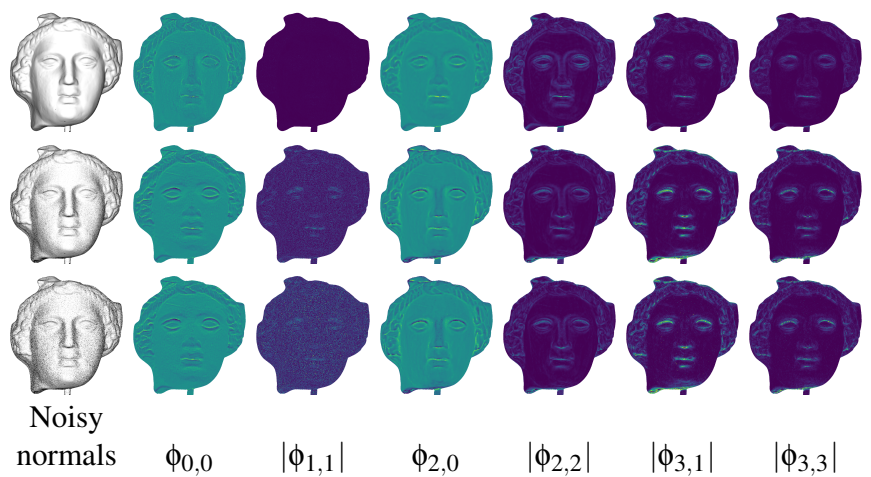

Figure 4: Evolution of the coefficients of a 3-Wavejets with increasing Gaussian noise on the normal direction (First row: no noise, 2nd row: Gaussian noise of standard deviation $\pi / 15,3 r d$ row: Gaussian noise of standard deviation $\pi / 9$ ). Notice how $\phi_{1,1}$, which is 0 without noise, captures most of the noise when the normals are perturbed. $\phi_{0,0} \neq 0$ because the order of a 3-Wavejets is too low to catch all the details of this shape given a large radius, leaving details as residue in $\phi_{0,0}$.

applying the normal enhancement filter, the positions of the surface points are unchanged. However since rendering algorithms are more sensitive to normals that positions, it looks as if the positions had been modified. Algorithm 2 sums up the normal enhancement filter.

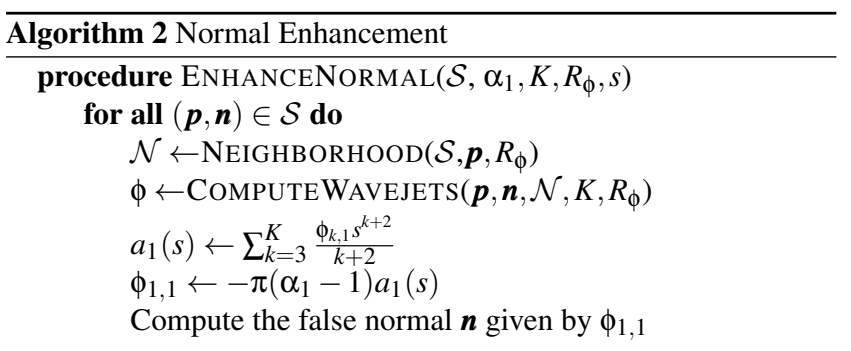

\section{Application to point sets}

\subsection{Wavejet Decomposition Equation}

Given a surface $\mathcal{S}$ that is only known through a set of measured points possibly spoiled by noise, we want to compute the Wavejets representation of the underlying surface up to a chosen order $K$, at an input point $\boldsymbol{p}$. Let us assume that the surface is locally sufficiently smooth, i.e. $\mathcal{C}^{K}$ in a neighborhood of radius $R_{\phi}$ around $p$. Our goal is to compute the $\phi_{k, n}$ coefficients that best decompose the underlying surface on the basis functions $B_{k, n}(r, \theta)=r^{k} e^{i n \theta}$ in the neighborhood $\mathcal{N}(p)$ of radius $R_{\phi}$ of $p$. Let $L$ denote the number of samples in this neighborhood, and let $q_{l}$ be one of these samples, with cylindrical coordinates $\left(r_{l}, \theta_{l}, z_{l}\right)$ w.r.t. an axis that corresponds to a rough approximation of the normal direction at point $p$. Then, the decomposition problem is formulated as finding $\phi_{k, n}$ minimizing:

$$
E(\Phi)=\sum_{l=1}^{L}\left\|z_{l}-\sum_{k=0}^{K-1} \sum_{n=-k}^{k} r_{l}{ }^{k} e^{i n \theta_{l}} \phi_{k, n}\right\|_{2}^{2}
$$


For clarity, we state the problem using the $\ell^{2}$ norm even if it is unreliable if there are outliers. When dealing with noisy point sets or outliers we solve this minimization using an iteratively reweighted least squares procedure. The weighting scheme involves the use of a diagonal matrix of weights $W$ that are used to leverage the importance of emerging outliers within the norm.

To reformulate this energy minimization, let us reorder the $B$ basis functions $B_{k, n}$ into a vector $V_{b}$, and the $B$ unknown $\phi_{k, n}$ into a vector $\Phi$, such that the $b^{\text {th }}$ component of $\Phi$ corresponds to the coefficient of the $b^{\text {th }}$ basis function in the decomposition. Let $k_{b}$ and $n_{b}$ respectively denote the order of derivation, and the oscillation frequency of the $b^{\text {th }}$ basis function $(0 \leq b \leq B-1)$. Using these notations, the energy to be minimized is the following:

$$
E(\Phi)=\sum_{l=1}^{L}\left(z_{l}-\sum_{b=1}^{B} r_{l}^{k_{b}} e^{i n_{b} \theta_{l}} \Phi_{b}\right)^{2}
$$

This amounts to the minimization of $\|M \Phi-Z\|_{2}^{2}$, where $Z$ is a vector of size $L$ containing the heights $z_{l}$ of neighbors $q_{l}$ and $M$ is a matrix of size $L \times B$ such that:

$$
M_{l, b}=r_{l}^{k_{b}} e^{i n_{b} \theta_{l}}
$$

Minimizing $\|M \Phi-Z\|_{2}^{2}$, is done by a $\mathrm{QR}$ decomposition of $M$. Thus computing the Wavejets decomposition around a point $\boldsymbol{p}$ amounts to building matrices $M$ and $Z$ and performing the QR decomposition of $M$. Using a Cholesky decomposition instead of QR fails because $M M^{*}$ is often ill-conditioned.

\subsection{Algorithm}

In order to compute Wavejets in the tangent plane, one can compute a first estimate of Wavejets in a parameterization plane close enough to the tangent plane. The initial parameterization plane is obtained through a Principal Component Analysis. Importantly enough the orientation is necessary neither to compute the Wavejets decomposition nor for the filtering, we only need a local parameterization with respect to an approximate tangent plane. Then the parameterization plane is corrected into the tangent plane using Corollary 1 and the Wavejets coefficients themselves are corrected using Corollary 2.

Given a point set of $N$ points, $K$ the Wavejets order and $L$ the number of neighbors, the complexity of the computation, using Equation 19 for all points is $O\left(N L K^{2}\right)$. To be able to solve the equation we pick $L \approx K^{2}$, yielding a final complexity of $O\left(N K^{4}\right)$. As a consequence, when $K$ is big (i.e. 13 for example), the computation cost increases a lot. Once the Wavejets decomposition is computed, the filtering amounts to computing a sum of $K$ terms for each point, because the filter only involves coefficients of frequency 0 or $1\left(K / 2\right.$ coefficients instead of $\left.K^{2}\right)$. Then each point is moved in its normal direction, which is constant in time, thus the filtering complexity reduces to $O(N K)$.

\section{Experiments and comparisons}

Our algorithm was implemented in C++ using Nanoflann and Eigen libraries, and parallelized using OpenMP. Detail enhancements is performed by first computing the Wavejets decomposition and then

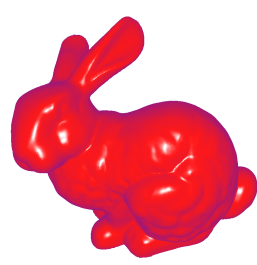

Original

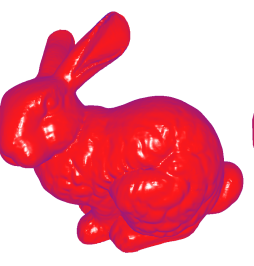

Enhanced normals Enhanced positions
Figure 5: Normal and position enhancement on a bunny with 6Wavejets. $R_{\phi}$ is equal to $3 \%$ of the shape diameter, and $\alpha_{0}=\alpha_{ \pm 1}=$ 2.
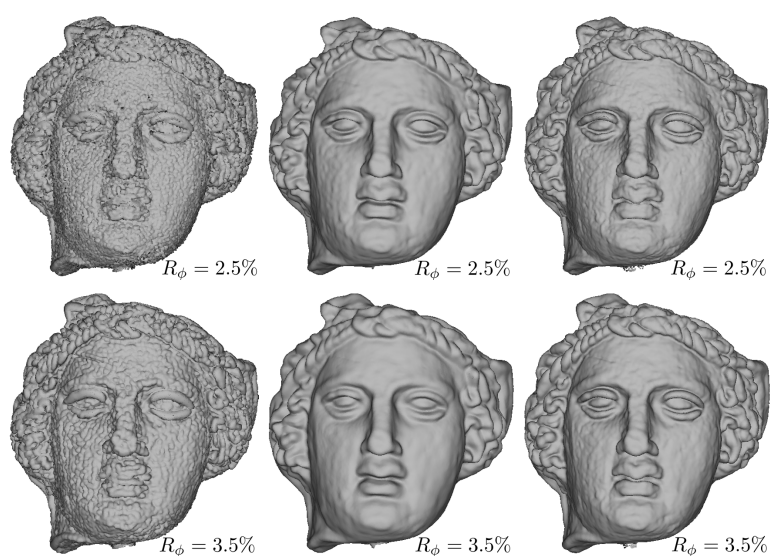

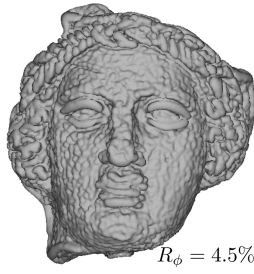

$K=8$

Unsharp Masking baseline

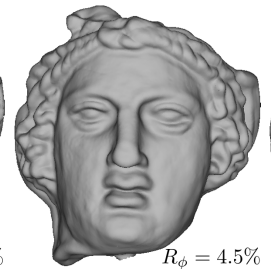

$K=2$

Unsharp Masking baseline

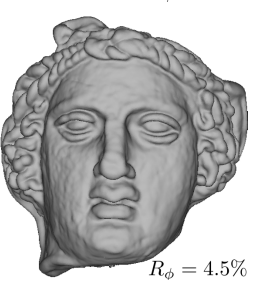

$K=8$

Ours
Figure 6: Detail enhancement for different $K$-Wavejets and radii. First column: naive Unsharp Masking with a precise mean curvature computed using 8-Wavejets. Unsharp Masking is unstable when applied to a precisely computed curvature. Second column: our position-based detail enhancement algorithm (2-Wavejets). This filtering is equivalent to a coarse Unsharp Masking since $a_{0}$ is proportional to the mean curvature when $K=2$. Third column: result for $K=8$. Increasing neighborhood radius makes the procedure less sensitive to small local variations while still catching larger details with a high resolution. $\alpha_{0}=3 / R_{\phi}$.

applying the detail enhancement filters. Table 1 gives the parameters and computation times for different models. The computational bottleneck lies in the Wavejets decomposition. Although the computation times are already good enough for processing common $3 \mathrm{D}$ point sets, the implementation could be made faster by porting the code to GPU.

In our implementation, for numerical reasons, the local surface is rescaled before computing the Wavejets decomposition so that 


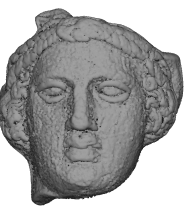

APSS

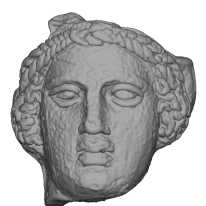

APSS

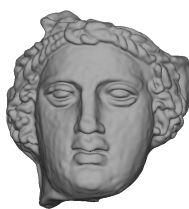

APSS

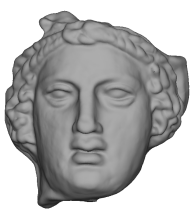

APSS

Scale 30
Figure 7: Unsharp Masking applied with mean curvature computed with APSS [GGG08] (Meshlab implementation) for different Minimum Least Squares (MLS) scales. MLS spherical parameter is set to 1. Each point is moved in the normal direction with a magnitude of 3 times the mean curvature

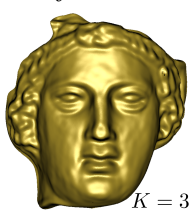

$\alpha_{ \pm 1}=2$

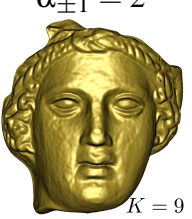

$\alpha_{ \pm 1}=2$

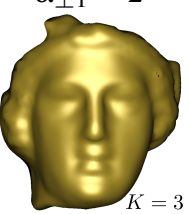

$\alpha_{ \pm 1}=0$

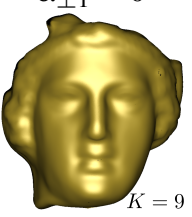

$\alpha_{ \pm 1}=0$

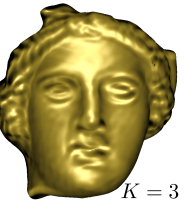

$\alpha_{ \pm 1}= \pm 2 \boldsymbol{i}$

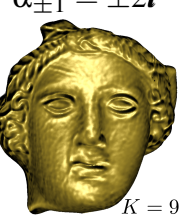

$\alpha_{ \pm 1}= \pm 2 i$

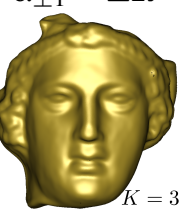

$\alpha_{ \pm 1}=1$

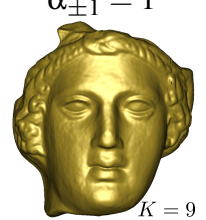

$\alpha_{ \pm 1}=1$

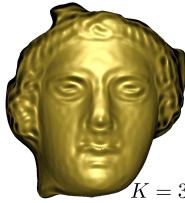

$\alpha_{ \pm 1}=-2$

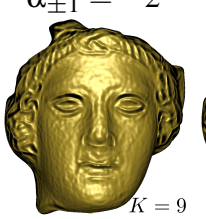

$\alpha_{+1}=-2$

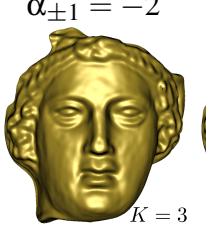

$\alpha_{ \pm 1}=2$

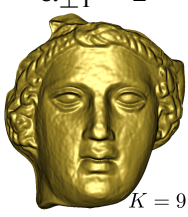

$\alpha_{ \pm 1}=2$

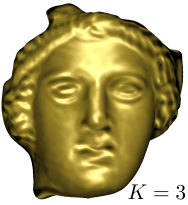

$\alpha_{ \pm 1}=\mp 2 i$

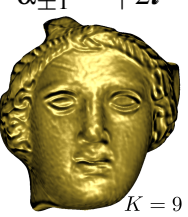

$\alpha_{ \pm 1}=\mp 2 i$

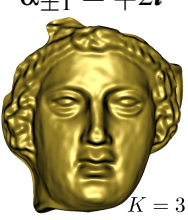

$\alpha_{ \pm 1}=4$

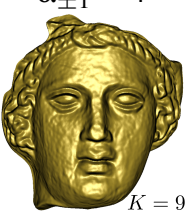

$\alpha_{ \pm 1}=4$
Figure 8: Influence of normal amplification gain $\alpha_{+1}$ and of order $K$. Similarly to Figure 6, a high order yields a fine enhancement. The phase of $\alpha_{ \pm 1}$ sets the orientation followed by normal amplification. When $\alpha_{ \pm 1}=0$, the normals are blurred.

$R_{\phi}=1$. Sometimes, locally, the surface does not project well on the tangent plane, this corresponds to locations where the surface cannot be expressed as a height field over the parameterization plane in a neighborhood of radius $R_{\phi}$, but it would project nicely if the radius was smaller. This happens for example on the fingers of Armadillo on Figure 13. In such situations, Wavejets tend to have high amplitudes and so do the $a_{n}$ coefficients, leading to high amplitude motion. To alleviate this problem, we set a threshold on the motion amplitude. In our experiments this threshold is set to $\frac{R_{\phi}}{2}$. In all our experiments we set the radius $s$ for computing $a_{0}$ and $a_{1}$ equals $R_{\phi}$.

The input to our algorithm is a point set with coarse normals which can be computed with a Principal Component Analysis. If the normal orientation is provided, this information is used to re-

\begin{tabular}{|c|c|c|c|c|}
\hline Shape & $N$ & Order & $\begin{array}{c}\text { Decomposition } \\
\text { time }\end{array}$ & $\begin{array}{c}\text { Filter } \\
\text { time }\end{array}$ \\
\hline Armadillo & $5 M$ & $K=6$ & $11 \mathrm{~min} 31 \mathrm{~s}$ & $1.7 \mathrm{~s}$ \\
\hline Bunny & $600 k$ & $K=6$ & $49 \mathrm{~s}$ & $187 \mathrm{~ms}$ \\
\hline Caesar & $600 k$ & $K=8$ & $2 \mathrm{~min} 18 \mathrm{~s}$ & $200 \mathrm{~ms}$ \\
\hline Pyramid & $1.5 M$ & $K=6$ & $1 \mathrm{~min} 43 \mathrm{~s}$ & $614 \mathrm{~ms}$ \\
\hline Manuscript & $1.5 M$ & $K=5$ & $1 \mathrm{~min} 44 \mathrm{~s}$ & $582 \mathrm{~ms}$ \\
\hline
\end{tabular}

Table 1: Computation times for various point sets and Wavejets orders. $N$ is the number of points (desktop computer with 2 Intel Xeon E5-2623 of 3.00GHz processors and 32GB RAM).

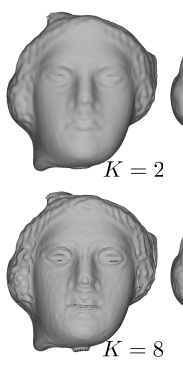

$\alpha_{0}=-2$
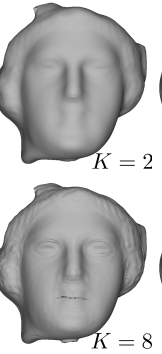

$\alpha_{0}=-1$
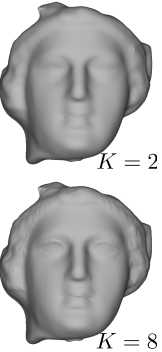

$\alpha_{0}=0$

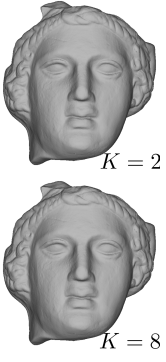

$\alpha_{0}=1$
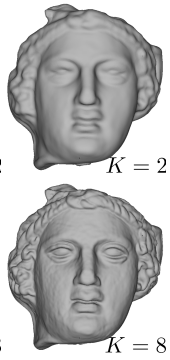

$\alpha_{0}=2$
Figure 9: Influence of position amplification gain $\alpha_{0}$ and order $K$. A lower order induces a coarser amplification. When $\alpha_{0}<0$, the shape tends to be carved in the orientation opposite to the details (visually similar to normal filtering with $\alpha_{ \pm 1}<0$, Fig. 8).

strict the neighborhoods to points having normals deviating up to $\frac{\pi}{2}$ with regard to the normal of the center point. This is done to avoid capturing two different surface sheets in a neighborhood.

The stability of the Wavejets coefficients w.r.t. normal error is shown in Figure 4. As can be seen, adding noise on the input normal direction mostly impacts $\phi_{1, \pm 1}$, while order 2 coefficients are very stable, which confirms the results of Theorem 1 . In order to test the stability of the $a_{0}$ coefficients, the position enhancement filter was tested on a perfect sphere of radius 1 randomly sampled (600k points). The parameters for the filter were $R_{\phi}=s=0.03$, $K=8$ and $\alpha_{0}=100$. The resulting shape is, unsurprisingly, a sphere with a larger radius. The radius of this enhanced sphere, computed by measuring the average distance to the center, is 1.07 with a standard deviation $\sigma=3 \cdot 8 \cdot 10^{-5}$, meaning that our computation is quite stable. The resulting sphere is smooth and does not exhibit any bumps. The theoretical equivalence between Wavejets and the Zernike polynomials was also tested numerically. Given a Wavejets and a Zernike decomposition of the same surface w.r.t. the same parameterization plane, the average distance between the 2 reconstructed surfaces is around $10^{-15}$, i.e. of the order of numerical precision.

Figure 5 shows the effects of normal and position based detail enhancement filters on the Bunny. The exaggerated features have a large scale, for example the borders of the ears and the fur features. On Figure 6, one can see the influence of the chosen order $K$ on the detail amplification. As the neighborhood radius increases, high order Wavejets allow to still amplify fine details, whereas low 


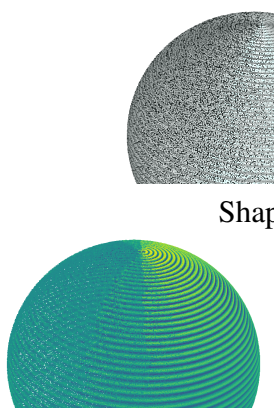

Wavejets
APSS, scale 2

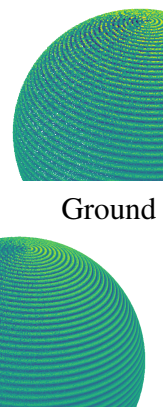

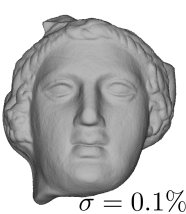
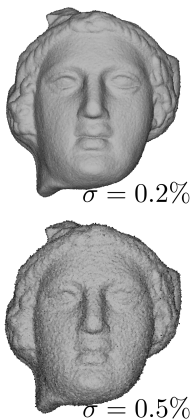

Input
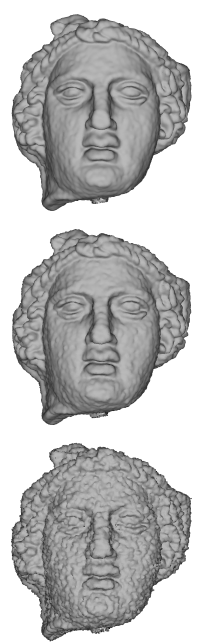

$\alpha_{0}=2$
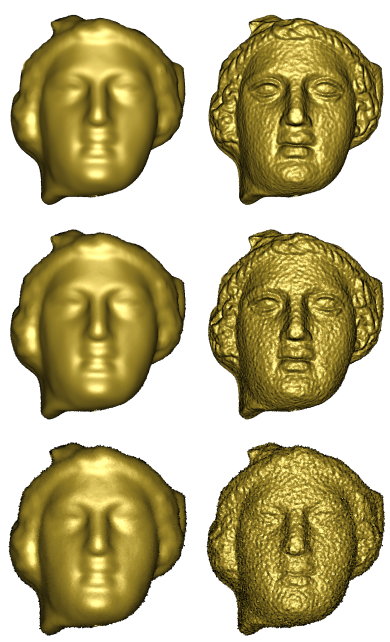

$\alpha_{ \pm 1}=0$

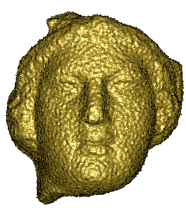

$\alpha_{ \pm 1}=2$ tation, spherical parameter set to 1$)$. The equation of the shape
is $\rho(\theta, \varphi)=1+0.005 \cos \left(100 \varphi^{2} / \pi\right)$ in spherical coordinates. In sparse areas (left part of the shape), our method tends to underperform (due to the necessary order change), similarly to APSS (scale 4). However, APSS (scale 2) performs well in sparse regions but is less accurate in dense regions than ours (right part of the shape).

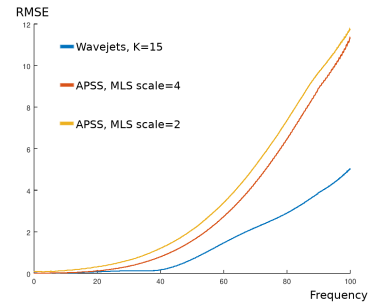

Dense

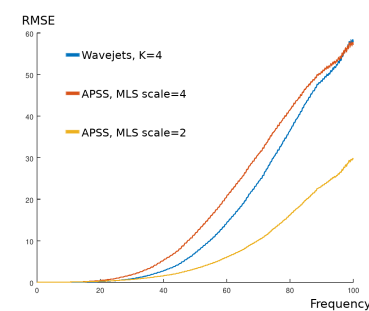

Sparse
Figure 11: Root Mean Square Error (RMSE) of estimated mean curvature with Wavejets and APSS [GGG08] on the shape of Figure 10. The left chart shows the RMSE computed on the part of the shape where the number of neighbors is stricly greater than 150 so $K=15$. The right chart shows the RMSE computed where the number of neighbors is between 17 and 31. Wavejets of order 4 are computed in such cases. $R_{\Phi}$ is $2.5 \%$ of the diameter of the shape.

order Wavejets tend to blur the shape. Since high order Wavejets give similar results over radius variation, a large neighborhood can be chosen to enhance details while being resilient to position noise. This holds as long as the height field assumption is respected. Figure 8 shows the influence of $K$ on normal filtering. The higher $K$, the more precise the output is. One can note that applying a normal enhancement procedure with $\alpha_{ \pm 1}=0$ cancels local normal dynamics. After normal update, $a_{ \pm 1}$ is close to 0 , which means that local normals dynamics become low. Setting $\alpha_{ \pm 1} \in \mathbb{C}$ locally twists normals (see Figure 15) and gives a twisted look to local features. The nose and lips of the mask statue are good examples of this twist (first and second rows of Figure 8). Similarly, Figure 9 shows the influence of $K$ and $\alpha_{0}$ on position enhancement. Setting $\alpha_{0}<0$ tends to carve the shape in the opposite direction of the details. Note that $\alpha_{0}=-2$ gives similar visual results as $\alpha_{ \pm 1}=-2$ (see Figure 8 and 9).
Figure 12: Influence of noise on detail enhancement: Noise was added to the positions of the input points. $R_{\phi}$ corresponds to $4.5 \%$ of the shape diameter. Position filtering with $\alpha_{ \pm 1}=0$ gives very stable results. Position enhancement has been performed using preprocessed normals filtered with $\alpha_{ \pm 1}=0$ so the direction of points motion remains stable over the shape.

We compare our method to a baseline high boost filter, by computing the mean curvature using Wavejets, and moving each point in a direction opposed to the normal direction at a rate equal to the mean curvature (Figure 6, first column). This naive amplification is very sensitive to noise or to small local variations because of the high resolution of the mean curvature computed with a high order $K$. In figure 7, we show Unsharp Masking applied to the mean curvature computed with Algebraic Point Set Surfaces (APSS) [GGG08] for different parameters. For high scales, the filter tends to produce similar outputs as our method with a low order $K$. For a low scale, it tends to enhance very small variations. There is a range of scales in which it produces similar results as our implementation using $a_{0}$ or a low order $K$ with a small radius (see Figures 6 and 7). In Figure 10, we show how Wavejets handle high curvature variations and sparsity compared to APSS for computing mean curvature. We use the PCA regression plane as parameterization plane with $R_{\phi}$ as the neighborhood radius. Figure 11 shows the Root Mean Square Error of computed mean curvatures. In dense regions, our estimate starts to fail after frequency 40 . Our representation approximates the surface with high precision in dense regions, yielding lower RMSE than APSS. As sparsity increases, Wavejets order decreases, yielding a coarser surface approximation. As one can visualize in Figure 10, APSS performs better than our method in such cases with MLS scale set to 2 .

Detail exaggeration filters are more likely to be sensitive to position noise. If noise is considered as a detail to amplify by the algorithm, the output will tend to be noisier than the input. Our algorithm is resilient until a certain amplitude of noise. If the neighborhood radius $R_{\phi}$ is large enough, noisy fluctuations in the signal tend to compensate over the radial integration. On Figure 12, different amplitudes of artificial noise over a shape are shown. On Figure 13 , the detail exaggeration filter on the position is applied to the 

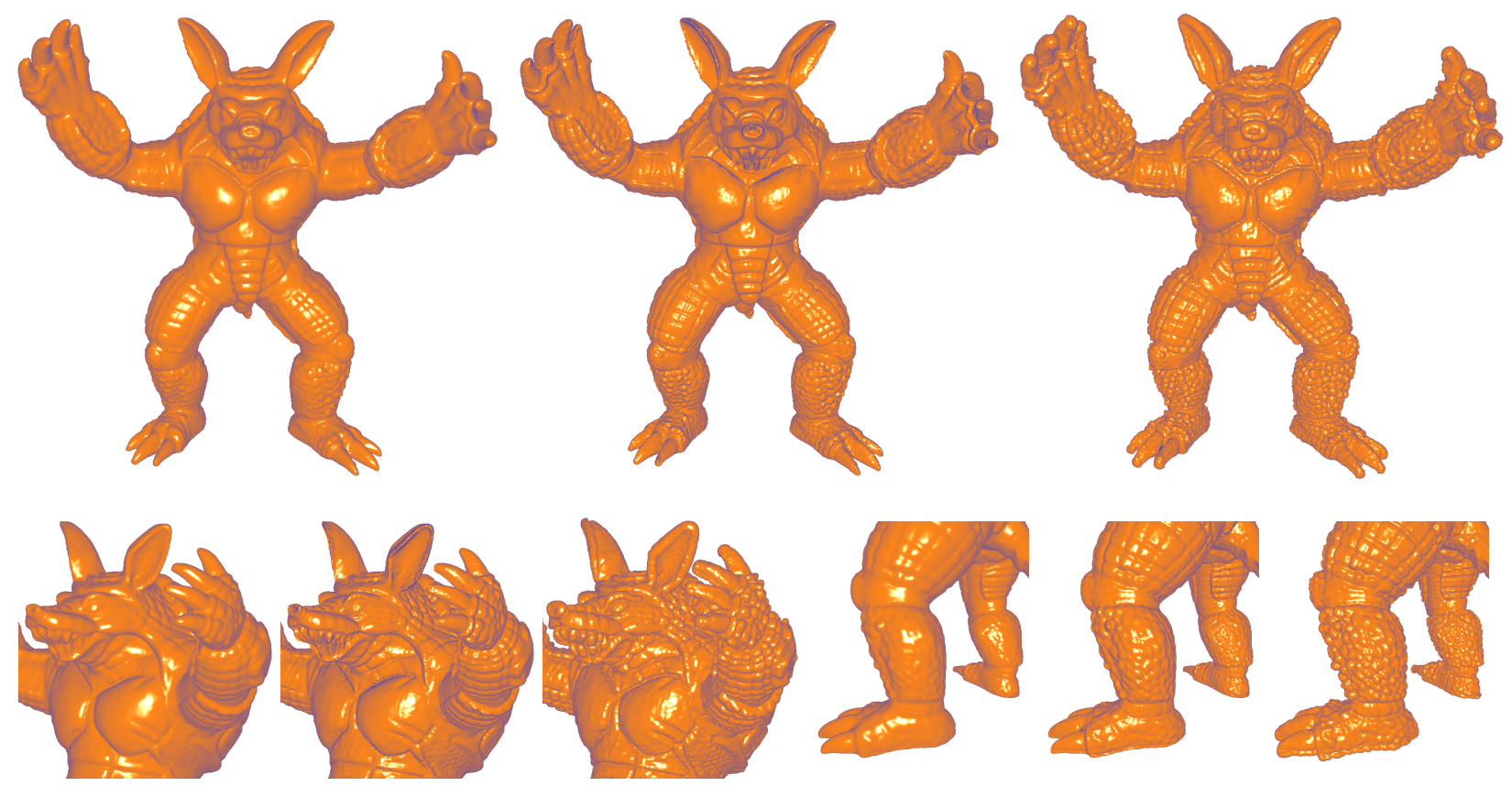

Figure 13: Outputs of our procedures on an armadillo (Left:original; Middle: normal-based detail enhancement with $K=7, \alpha_{ \pm 1}=3 ;$ Right: position-based detail enhancement with $K=6, \alpha_{0}=2$ ).

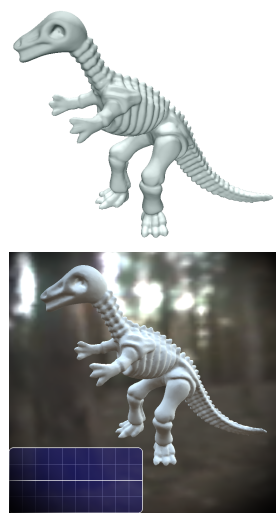

Original
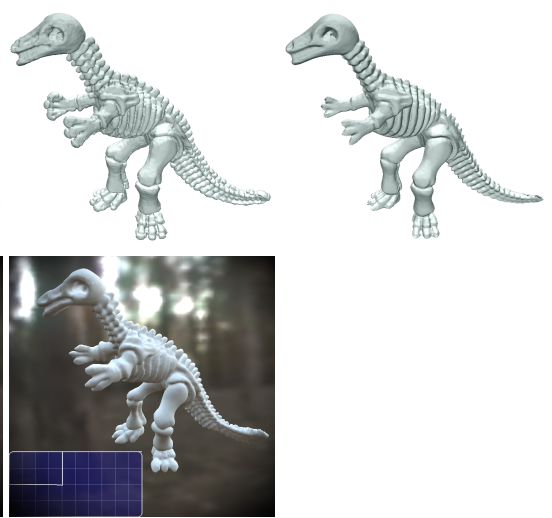

Positions enhanced Normals enhanced

Figure 14: On the left: input shape. On the top right: our position and normal enhancement method. On the bottom right: the result of a high-boost filter applied to manifold harmonics of the dino (no normal enhancement is defined using this method). Our position enhancement method highlights more local details as the ManifoldHarmonics based high-boost filter. The rendering of the second row is a capture of the MHB demo provided by the authors.

Armadillo shape. The details are particularly well enhanced by the Wavejets filter: creases are more distinctive and small details, such as the tortoise-shell texture on the legs, are amplified. The eyebrow and teeth are also more prominent.

Figure 14 shows the difference of the output of our position filter with a high-boost filter applied to manifold harmonics. One can see that our result enhances the details in a different manner: the
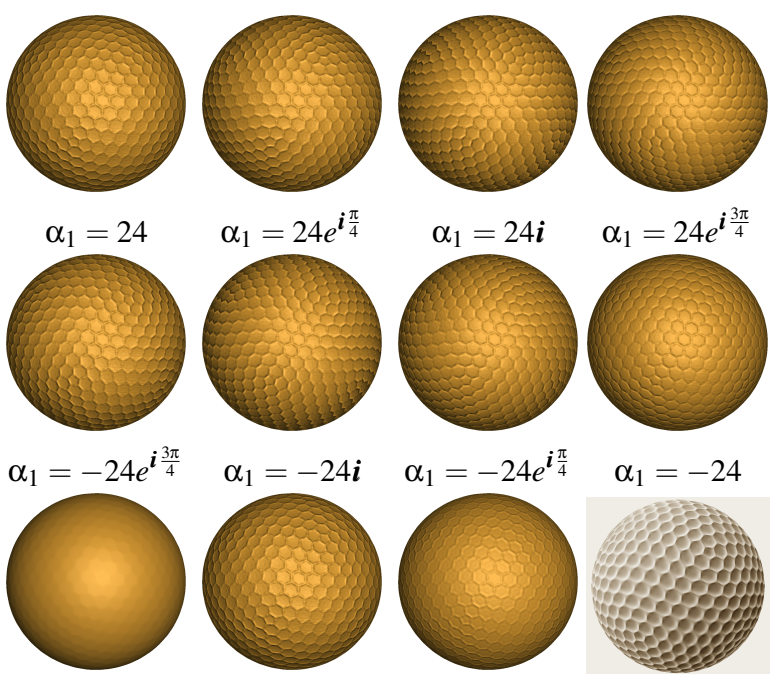

Original

Ours $\left(\alpha_{1}=24\right)$

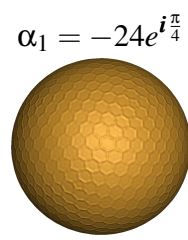

[CST05] $\alpha_{1}=-24$

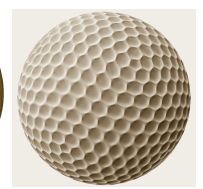

[RBD06]
Figure 15: Normal enhancement on a golf ball. First and Second row : normal amplification for different $\alpha_{1}$ on a 9-Wavejet. Note that $\alpha_{-1}=\alpha_{1}^{*}$ on every examples. Last row: comparison with [CST05] normal enhancement algorithm and with [RBD06] detail exaggerating shading.

behavior highlighted by our approach is more local and less influenced by global shape structure. Indeed, both methods are very different, since Wavejets filters operate only locally while Manifold Harmonics take the whole shape into consideration for building the function basis. Furthermore, manifold harmonics often require a mesh (although some point clouds generalization exist). The man- 
ifold harmonics basis does not permit to filter or twist the normals in such a simple way as our method does.

On Figure 16, we compare the result of our normal filter with [CST05] and [RBD06]. [CST05] has an effect on the normals that is similar to our algorithm. This algorithm takes as input a neighborhood radius, a number of iterations to perform, and a step. If the number of iterations is too high, the normal enhancement [CST05] becomes unstable. On the contrary, our algorithm can perform higher detail enhancement while remaining stable. [RBD06] has a very different approach that is not based on enhancing normals, but rather on a shading algorithm taking the light direction into account in a multiscale way. Our Enhancement filters can also be used to exaggerate narrow details as shown in Figures 17 and 18 on archaeological artifacts. It makes details appear more clearly than on the original point set.

Parameters. The parameters for our algorithm are the following: the radius $s$ for the computation of the volumes $a_{0}$ and $a_{1}$ and $R_{\phi}$, which we set equal in our tests, the order for the Taylor Expansion $K$, and amplification gains $\alpha_{0}$ or $\alpha_{ \pm 1}$ depending on which filter is chosen. Note that $s=R_{\phi}$ should be chosen so that the number of neighbors $L$ of each point is above $\frac{(K+1)(K+2)}{2}$, which is the number of Wavejets coefficients of order $K$. The neighborhood size $L$ is an important parameter. If $L<\frac{(K+1)(K+2)}{2}$, the system to solve is underdetermined. In such cases, the order $K$ is locally decreased until the system can be safely solved. To do so, we add a parameter $\beta>1$, controlling the decrease rate of the order: $K$ is decreased until $L<\beta \frac{(K+1)(K+2)}{2}$. We set $\beta=1.1$ in our implementation. In presence of noise, $\beta$ should be greater.

Limitations Our method is still computationally expensive. The computational bottleneck lies in the system solve in order to compute the Wavejets for each point, with a complexity of $O\left(N K^{4}\right)$. The Wavejets order choice is thus critical for the computation time, which is coherent with the measures of Table 1. In the presence of fast enough varying features, stability of Wavejets coefficients decreases (see figure 11). Another limitation of our algorithm lies in the assumption that the surface can be expressed as a height field over a parameterization plane inside a neighborhood of given radius $r$ constant over the surface. For some points, $r$ might be too large for the assumption to hold and spurious artifacts can appear. To alleviate this effect, Hamdi-Cherif et al. [HCDC17] proposed to encode the surface as a height field over a quadric. This simple idea would however require using the Fourier transform on geodesic circles, which is a nontrivial adaptation. Another solution could be to adapt the radius to the local feature size. [MGB $\left.{ }^{*} 12\right]$ proposes an interesting approach to solve the problem of the right scale estimation that could be adapted to Wavejets as a future work. As an alternative, using multiscale Wavejets could alleviate the scale choice.

\section{Conclusion}

We introduced a new basis for decomposing locally a surface in a basis that emphasizes both angular oscillations and surface derivatives. This decomposition is proven to be stable with respect to a small rotation of the parameterization plane from the tangent plane. This allows to define and compute surface characteristic functions at a very high precision and build explicit shape enhancing filters, acting either on the normals or the point positions with a view of amplifying, modifying or inverting shape details. Wavejets can have a broader interest than this application, in a future work we plan to study its application to surface description for shape matching and shape registration.

Acknowledgements This work was supported by the ANR PAPS project, grant ANR-14-CE27-0003 of the French Agence Nationale de la Recherche.

\section{References}

[CP03] Cazals F., Pouget M.: Estimating differential quantities using polynomial fitting of osculating jets. In Proc. SGP '03 (2003), Eurographics, pp. 177-187. 1, 2, 4, 5

[CST05] Cignoni P., Scopigno R., TARini M.: A simple normal enhancement technique for interactive non-photorealistic renderings. Computers \& Graphics 29, 1 (2005), 125 - 133. 2, 10, 11, 12

[DM14] Digne J., MOREL J.-M.: Numerical analysis of differential operators on raw point clouds. Numerische Mathematik 127, 2 (2014), 255-289. 2, 5

[DMIF15] Dekel T., Michaeli T., Irani M., Freeman W. T.: Revealing and modifying non-local variations in a single image. $A C M$ Trans. Graph. 34, 6 (Oct. 2015), 227:1-227:11. 2

[Gab65] GABOR D.: Information theory in electron microscopy. Laboratory Investigation 14 (1965), 801-807. 2

[GGG08] Guennebaud G., Germann M., Gross M.: Dynamic sampling and rendering of algebraic point set surfaces. Computer Graphics Forum 27, 2 (2008), 653-662. 2, 8, 9

[HCDC17] Hamdi-Cherif A., Digne J., Chaine R.: Superresolution of point set surfaces using local similarities. Computer Graphics Forum (2017). 11

[JS10] JoshI P., SÉQUIN C.: An intuitive explanation of third-order surface behavior. Comput. Aided Geom. Des. 27, 2 (Feb. 2010). 2

[KFR03] KaZhdan M., Funkhouser T., Rusinkiewicz S.: Rotation invariant spherical harmonic representation of $3 \mathrm{~d}$ shape descriptors In Proc. Symposium on Geometry Processing (2003), pp. 156-164. 2

[KH90] KHOTANZAD A., Hong Y. H.: Invariant image recognition by zernike moments. IEEE Transactions on Pattern Analysis and Machine Intelligence 12, 5 (May 1990), 489-497. 2

[LFB94] Lindenbaum M., Fischer M., Bruckstein A.: On Gabor's contribution to image enhancement. Pattern Recognition 27 (1994), 1-8. 2

[LSW09] LuO C., SAFA I., WANG Y.: Approximating Gradients for Meshes and Point Clouds via Diffusion Metric. Computer Graphics Forum (2009). 2

[LTF*05] LiU C., Torralba A., Freeman W. T., Durand F., ADELSON E. H.: Motion magnification. ACM Trans. Graph. 24, 3 (July 2005), 519-526. 2

[MCAG08] Mousa M., Chaine R., Akkouche S., Galin E.: Toward an efficient triangle-based spherical harmonics representation of 3D objects. Computer Aided Geometric Design 25 (2008), 561-575. 2

[MCH*06] ManaY S., CRemers D., Hong B.-W., YezZI A. SoAtTo S.: Integral Invariants and Shape Matching. Birkhäuser Boston, Boston, MA, 2006, pp. 137-166. 2, 5

[MDSB03] Meyer M., Desbrun M., Schröder P., BARr A. H.: Discrete Differential-Geometry Operators for Triangulated 2-Manifolds. Springer Berlin Heidelberg, Berlin, Heidelberg, 2003, pp. 35-57. 2

[MGB*12] Mellado N., Guennebaud G., Barla P., Reuter P., SCHLICK C.: Growing least squares for the analysis of manifolds in scale-space. Comput. Graph. Forum 31, 5 (Aug. 2012), 1691-1701. 11 


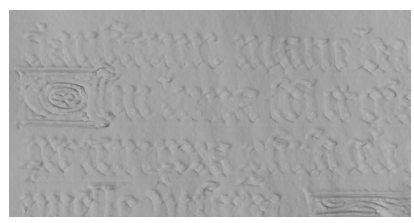

Original

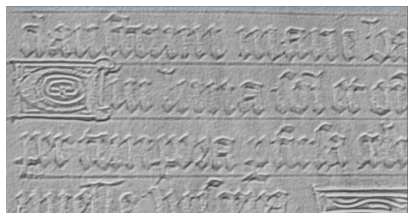

Enhanced shader [RBD06]

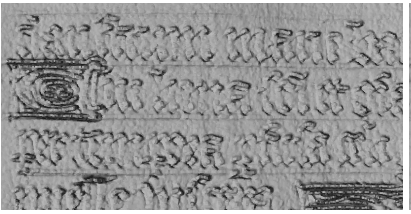

[CST05]

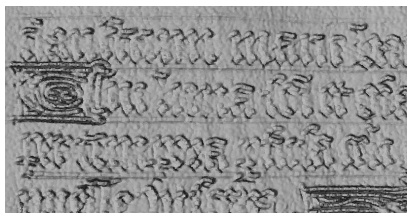

Ours $\left(K=5, \alpha_{ \pm 1}=3\right)$

Figure 16: Normal enhancement with different methods. Note that our algorithm has very similar results as [CST05] (parameters: are a step of 0.1 with 30 iterations). The main difference between the two procedures is that ours is not iterative, and might be more straightforward for parameters choice. [RBD06] gives better visual results for this case, but is a totally different method which relies on a mesh where we use unstructured point sets. Each point is rendered through a shading procedure taking the light direction into account while we only rely on the local geometry of the surface. The snapshot of [RBD06] was done using Xshade, a software provided by the authors.
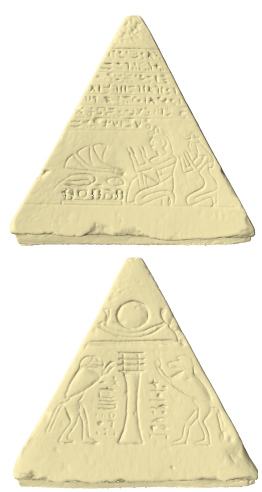

Original

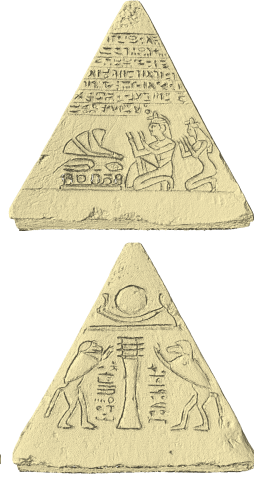

Normal enhanced
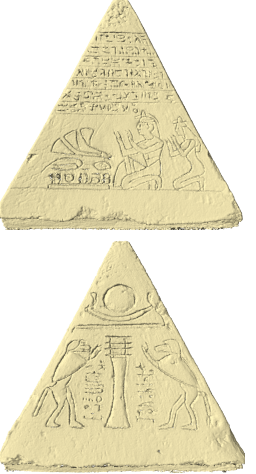

Position enhanced

Figure 17: Applying order 7 (normal filter) and order 6 filters (position filter) to the Pyramid datasets with $\alpha_{0}=\alpha \pm 1=2$.
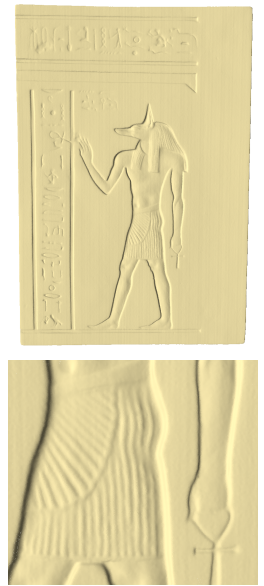

Original
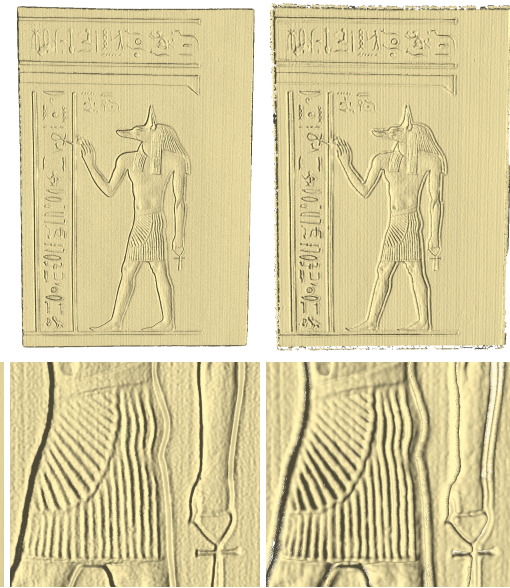

Normal enhanced Position enhanced

Figure 18: Applying order 9 (normal exaggeration) and order 8 filters (position filter) to the Anubis datasets with $\alpha_{0}=\alpha_{ \pm 1}=2$.

[MLLY91] Mitra S. K., Li H., LiN I. S., YU T. H.: A new class of nonlinear filters for image enhancement. In Proc. ICASSP (1991), pp. $2525-2528$ vol. 4 . 2

[MT98] Mehlum E., TARRou C.: Invariant smoothness measures for surfaces. Advances in Computational Mathematics 8-1 (1998), 49-63. 2

[NK03] Novotni M., KLEIN R.: 3d zernike descriptors for content based shape retrieval. In Proceedings of the Eighth ACM Symposium on Solid Modeling and Applications (New York, NY, USA, 2003), SM '03, ACM, pp. 216-225. 2

[PG01] Pauly M., Gross M.: Spectral processing of point-sampled geometry. In Proc. Siggraph 2001 (2001), ACM, pp. 379-386. 2

[PKG06] Pauly M., Kobbelt L. P., Gross M.: Point-based multiscale surface representation. ACM Trans. Graph. 25, 2 (Apr. 2006), 177-193. 2

[PRM00] Polesel A., RAMPoni G., Mathews V. J.: Image enhancement via adaptive unsharp masking. IEEE Transactions on Image Processing 9, 3 (Mar 2000), 505-510. 2

[PWHY09] Pottmann H., Wallner J., Huang Q.-X., Yang Y.L.: Integral invariants for robust geometry processing. Comput. Aided Geom. Des. 26, 1 (Jan. 2009), 37-60. 2

[PWY*07] Pottmann H., Wallner J., Yang Y.-L., LaI Y.-K., Hu S.-M.: Principal curvatures from the integral invariant viewpoint. $C A G D$ 24, 8-9 (2007), 428-442. 2, 5

[RBD06] Rusinkiewicz S., Burns M., DeCARlo D.: Exaggerated shading for depicting shape and detail. ACM Trans. Graph. 25, 3 (July 2006), 1199-1205. 2, 10, 11, 12

[RSKMY96] Ramponi G. G., Strobel N., K. Mitra S., Yu T.-H Nonlinear unsharp masking methods for image contrast enhancement. $J$. Electronic Imaging 5 (07 1996), 353-366. 2

[Tau95] TAUBIN G.: A signal processing approach to fair surface design. In Proc. Siggraph 95 (1995), ACM, pp. 351-358. 2

[TZG96] TAUBIN G., ZHANG T., GoluB G.: Optimal surface smoothing as filter design. Springer Berlin Heidelberg, Berlin, Heidelberg, 1996, pp. 283-292. 2

[VL08] VAllet B., LeVy B.: Spectral Geometry Processing with Manifold Harmonics. Computer Graphics Forum (2008). 2

[VPB*09] Vergne R., Pacanowski R., Barla P., Granier X., SCHLICK C.: Light warping for enhanced surface depiction. ACM Trans. Graph. 28, 3 (July 2009), 25:1-25:8. 2

[WMKG07] WARDETZKY M., MATHUR S., KÄLBERER F., GRINSPUN E.: Discrete laplace operators: No free lunch. In Proc. SGP 2007 (2007), pp. 33-37. 2

[ZBS04] ZHOU K., BAO H., SHI J.: 3d surface filtering using spherical harmonics. Computer-Aided Design 36, 4 (2004), 363 - 375. 2

[Zer34] ZERNIKE V. F.: Beugungstheorie des schneidenver-fahrens und seiner verbesserten form, der phasenkontrastmethode. Physica 1 (May 1934), 689-704. 2, 4 